\title{
The Law of Non-Contradiction as a Metaphysical Principle
}

\author{
Tuomas E. Tahko \\ DURHAM UNIVERSITY \\ HTTP://WWW. TTAHKO.NET/ \\ tuomas.tahko@durham.ac.uk \\ Received by Greg Restall \\ Published June II, 2009 \\ http://www.philosophy.unimelb.edu.au/ajl/2009 \\ (C) 2009 Tuomas E. Tahko
}

\begin{abstract}
The goals of this paper are two-fold: I wish to clarify the Aristotelian conception of the law of non-contradiction as a metaphysical rather than a semantic or logical principle, and to defend the truth of the principle in this sense. First I will explain what it in fact means that the law of non-contradiction is a metaphysical principle. The core idea is that the law of non-contradiction is a general principle derived from how things are in the world. For example, there are certain constraints as to what kind of properties an object can have, and especially: some of these properties are mutually exclusive. Given this characterisation, I will advance to examine what kind of challenges the law of noncontradiction faces-the main opponent here is Graham Priest. I will consider these challenges and conclude that they do not threaten the truth of the law of non-contradiction understood as a metaphysical principle.
\end{abstract}

\section{INTRODUCTION}

The purpose of this paper is to defend the idea that the law of non-contradiction (LNC) is a metaphysical rather than a logical principle I will also defend the status of LNC as the best candidate for a fundamental metaphysical principle-if there are any principles which constrain the structure of reality, then LNC is certainly our most likely candidate. ${ }^{2}$ Some challenges to this view

'The idea has its roots in Aristotle's Metaphysics, see also Politis (2004: ch. 5). It should perhaps be noted here that although I aim to show that LNC is a metaphysical rather than a logical principle, we are nevertheless dealing with an important topic in philosophical logic. If I am right, the status of LNC has ramifications towards the nature of logic in general. It may be that other, or even all logical laws can be interpreted in a metaphysical fashion; this would require us to re-evaluate the metaphysical status of logic.

${ }^{2}$ I will not discuss the law of the excluded middle here, which is perhaps another likely candidate for a fundamental metaphysical principle. It appears that for Aristotle at least, LNC was always the more primary of the two principles, as he says that LNC is 'the most certain of 
will be considered, namely those suggested by Graham Priest. There is abundant contemporary literature about what LNC is and how one might defend or reject it 3 , but the majority of the discussion concerns the logical or semantic reading of LNC. Here I will analyse the principle from a strictly metaphysical point of view and suggest some guidelines as to how one might go on about defending or rejecting the principle in this sense, as well as consider what kind of implications this has for the use of LNC in logic.

Before we can advance any further, it must be settled which formulation of LNC we wish to use. For my purposes, the typical formulation 'not both $\mathrm{P}$ and not-P', is unsatisfactory, although we could perhaps arrive at the same conclusion by starting from this formulation. We will, however, be better off with one of Aristotle's many formulations of LNC, such as 'the same attribute cannot at the same time belong and not belong to the same subject in the same respect' (Aristotle 1984: I005br9-20). When put like this, the principle appears considerably deeper, as it clearly states a restriction that concerns things rather than just propositions. We can thus avoid a prolonged discussion about the nature of propositions. We might very well be able to extend the applicability of LNC beyond the ascriptions of properties to objects, but for the sake of simplicity we will only discuss this version here. The semantic or logical interpretation of LNC, which we aim to undermine, considers LNC to be a principle that governs our thoughts and perhaps our language, or even more weakly, simply a principle which is true in certain models (i.e. logical systems) and has no bearing on (mind-independent) reality.

\section{THE METAPHYSICAL INTERPRETATION OF LNC}

At its simplest, the metaphysical interpretation of LNC amounts to this: the entities of the mind-independent reality are plausibly governed by some sort of principles (as otherwise there would be no order in our experience of them), that is, there are some constraints as to what kind of properties a certain kind of entity can and cannot have, and further, some of these properties are mutually exclusive. For instance, a particle cannot both have and not have a charge at the same time, or an object cannot be both green and red all over at the same time. It seems that reality just $i s$ such that it conforms to the law of noncontradiction. Aristotle's different formulations of the law attempt to express this orderliness in a simple manner.

all principles' (Aristotle I984: IOO5b22). Furthermore, the law of the excluded middle can be derived from LNC (with the help of De Morgan's laws and the principle of double negation) and the latter is generally more widely accepted, e.g. LNC holds even in intuitionistic logic whereas the law of the excluded middle does not (the law of the excluded middle is not derivable from LNC in intuitionistic logic). All this seems to make LNC the likeliest candidate for a fundamental principle.

${ }^{3}$ Most notably Priest et al. (2004). 
An initial concern about the formulation of LNC should be addressed here: all formulations of LNC seem to rely on negation, and negation, being a logical connective, may seem out of place in a metaphysical formulation of LNC, 4 In fact, given an appropriate interpretation of negation, i.e. the so called paraconsistent negation, which is not explosive (cf. Slater 1995), even the opponents of LNC might be able to accommodate it. While this is an important point, I think that it does not do justice to the Aristotelian formulation of LNC, namely 'the same attribute cannot at the same time belong and not belong to the same subject in the same respect' (Aristotle 1984: 1005bi9-20). This formulation does indeed use negation, but the emphasis is on the mutual exclusiveness of having a certain attribute and lacking that attribute at the same time; indeed, the idea can be expressed without using negation at all: the mutual exclusiveness of certain properties is evident even without the concept of negation. In a manner that bears some similarity to what Price (1990) has suggested it will thus be possible to interpret negation in terms of mutual exclusiveness. Accordingly, LNC can be formulated in a thoroughly metaphysical sense. For the sake of brevity we will nevertheless continue to use negation in the examples that follow.

Another thing to note before we proceed is that semantic paradoxes such as the Liar do not threaten LNC as a metaphysical principle 5 Any arbitrariness or vagueness over language has no bearing on LNC understood as a metaphysical principle. A counterexample to the metaphysical version of LNC could only be a true contradiction in the world. But before we will consider such counterexamples, we must get to the bottom of what LNC in fact says.

Let us consider the example mentioned above: one and the same particle cannot both have and not have an electric charge at the same time. The concept of an electric charge is admittedly arbitrary, especially in the context of trying to define a fundamental metaphysical principle, but perhaps we can elaborate on this. We know that, for instance, electrons and protons have an electric charge of the same magnitude, but with the opposite polarities: electrons have a 'negative' charge and protons a 'positive' charge. Now, when we say that a particle cannot both have and not have a charge at the same time, we can think of this as a restriction in terms of the implications that an electric charge has. The most important of these implications is that like charges repel and unlike charges attract, and thus electric charges are responsible for one of the fundamental forces, namely the electromagnetic force. Setting aside for the moment what electric charges actually are, it seems that to produce the

${ }^{4}$ Thanks to an anonymous referee for this observation, as well as pointing towards a potential solution in the lines of Price (1990).

${ }_{5}$ Accordingly, I will not discuss the Liar or other semantic paradoxes here, however, see for instance Eklund (2002A, 2002B), for an account of inconsistency in language. The recent debate between Beall \& Priest (2007) and Eklund (2008), interesting as it is, is thus not directly relevant to the discussion at hand, although our discussion may very well have implications towards their debate as well. 
effects that they evidently do the one and the same particle must consistently either have an electric charge or not have an electric charge at any one time 6 If we think of the electric charge as a property of an entity, say an electron, it is a fully exhaustive property, as the charge can be of exactly one type and this exhausts any further qualifications. Of course, this is not to say that the charge could not change (both in strength and polarity), just that at any given time it must either be present or not. All of this, you might think, is obvious; it is obvious because we are used to things that conform to LNC. What is not obvious is why they do so.

The metaphysical reading of the law of non-contradiction suggests an answer to the question why our observations conform to the principle: because LNC is a true metaphysical principle concerning the world. Let us trace the route from our observations of the world to the mind-independent reality which supposedly conforms to LNC. Basically, you can insert any kind of metaphysically realist ontology here, it makes little difference for our purposes. What we need to agree about is that whatever the organisation of the entities in the world is, it does not violate LNC.

Our observations suggest that an electric charge is a property that an entity can have in two different varieties: the positive and the negative. What suggests that this is a universal (and actual) condition-apart from the fact that we have never observed an entity that both has and does not have a charge at the same time-is that the causal powers associated with electric charges could not arise if the same entity could both have and not have a charge at the same time. For instance, atoms would not hold together. Perhaps one might suggest that if the same entity both did and did not have a charge at the same time, it would follow that atoms both would hold and would not hold together. But how could this be the case? If atoms both would hold and would not hold together, then it seems that all macrophysical objects both would and would not exist. No: macrophysical existence requires stability, and atoms that both hold together and do not hold together would certainly be quite unstable. It might be that it is not a metaphysically necessary condition for the existence of macrophysical objects that electric charges have the particular causal powers that they do; however, all that matters here is that in the actual world electric charges have the particular causal powers that they have and these powers emerge because electric charges act consistently. In other words, in the actual world the laws of physics require that one and the same particle either has or does not have an electric charge at any one time-otherwise this particular macrophysical construction would not be possible. The law of non-contradiction, if it is true, is perhaps the most fundamental condition of this type.

\footnotetext{
${ }^{6}$ More specifically, particles must conform to the Pauli Exclusion Principle, which states that no two identical fermions can have the same quantum number at the same time. By consistency I mean simply that we do not have any mutually exclusive properties at hand, as a violation of the Pauli Exclusion Principle would imply.
} 
Let it be noted that the above example concerns all macrophysical objects, that is, we are dealing with the existence conditions of all macrophysical objects, and I wish to suggest that one of those preconditions is consistency. If we take this condition away, the result is trivialism, at least regarding the existence of macrophysical objects.

One concern that could be raised at this point is the fallibility of our empirical story about electric charges and the forming of atoms: we could have gotten the story wrong and in fact the forming of atoms might be compatible with violations of LNC. While this epistemic concern is valid, it seems no more threatening than any quite general sceptical worry: unless we have some good reasons to challenge the current understanding of how atoms form (as well as fundamental principles of physics such as the Pauli Exclusion Principle), then the sceptical point is moot.

There might still be a worry that even if our experience conforms to LNC, this tells us nothing about the world, but rather only about the concepts we use in describing it. But consider what would happen if there really were a fundamental discontinuity between the world and the concepts that we use in describing it, namely, if the world did not conform to LNC. How would we be able to express anything about the world if this were the case? Perhaps there is a sceptical worry here which cannot be overcome, but anyone who takes this path would be on a slippery slope towards solipsism: if the consistency of the world is only an illusion, then you cannot trust any of your interactions with it, including your interactions with other people. Surely this is an infeasible position. It is certainly more likely that the concepts we use in experiencing the world have developed through our interaction with the world and thus correspond with it at least to a moderate degree 7 Admittedly, no one holds the view that all contradictions are true, but rather just some of them, so the problem would then be restricted to the issues that these contradictions concern, but this hardly removes the problem.

One conclusion that we might be tempted to draw from the discussion above is that LNC is fundamentally connected with rationality. However, there has been some discussion about whether LNC is really a condition for rationality (cf. e.g. Dutilh Novaes 2008). The problem, it is suggested, is that classical logic is not an accurate model of our rationality; in fact we might very well be able to accommodate contradictions in our reasoning. Be that as it may, it is not clear how we could model rationality without LNC, and more importantly, there does not seem to be much evidence of the effectiveness of reasoning that does not conform to LNC. The only possible example that I can think of is quantum mechanics, but everyone is on a very shaky ground here, as we will observe in section 6. Accordingly, the fact that we might be able to accom-

\footnotetext{
${ }^{7}$ The problem might not present itself in as strong a form as this for any actual opponents of LNC, but it may be that there is a danger of collapsing to trivialism lurking here, in which case the slippery slope would be valid.
} 
modate contradictions in our reasoning does not mean that we should: we are able to conceive of all sorts of things, even metaphysically impossible things, but not all of them have any bearing on reality. If the opponent of LNC can demonstrate that by adopting inconsistent reasoning we can model reality better than with consistent reasoning, then we might have to reconsider, but so far no plausible arguments that support this conclusion have been presented, as we will see in the course of this paper.

Finally, it is worth noting that even the main opponents of LNC have previously acknowledged that the observable world appears to be consistent (cf. Priest 1999, Beall 2000). Although there are a number of ways that we can have the illusion of observing a contradiction, such as in the case of the impossible figures familiar from the art of Maurits Escher, these hardly imply that there would be true contradictions in the world. Having said that, Beall and Colyvan (200I) have hinted towards a possible case for observable contradictions: if we adopt a paraconsistent account of vagueness and admit that some of the predicates that we use in scientific language are indeed vague, we would have a case for observable contradictions. This idea has been further developed in Hyde and Colyvan (2008). I do not have the space here to compare different approaches to vagueness, which would be necessary to thoroughly evaluate this claim, but it would appear that here as well, as in the case of the Liar paradox, the focus is on what Edwin Mares (2004) has dubbed semantic dialetheism 8 , namely the idea that there may be indeterminacy in semantics, but this does not imply that there has to be indeterminacy in the world. Thus Hyde and Colyvan insist that 'Our semantics of vagueness might admit of inconsistency without the world being thereby taken as inconsistent' (2008: II5). This may indeed be the case, but the purpose of this paper is to evaluate the thesis of metaphysical dialetheism, namely the idea that there are true contradictions in the world. In the next section we will examine the distinction between semantic and metaphysical dialetheism and consider how true contradictions in the world could be accommodated.

\section{AN ONTOLOGY OF CONTRADICTIONS?}

Given the metaphysical understanding of $\mathrm{LNC}$, let us see if it is possible accommodate violations of the principle in our ontology. There have been at least half-hearted attempts to do this.9 This is not a very typical topic in the dialetheist literature, as most of it is concerned with semantic paradoxes, which are not at issue here. I have no quarrel with the idea that there could be contradictions in language, as the Liar and other paradoxes would suggest. The issue at hand here, however, concerns whether dialetheism could be true in a metaphysically deep sense, that is, whether there could be contradictions in

\footnotetext{
${ }^{8}$ Dialetheism is the view that there is a proposition $P$ such that both $P$ and not- $P$ are true, i.e. there are some contradictions that are true.

9See Priest (2006: 300) and Beall (200ов, 2004).
} 
the world, in addition to the apparent linguistic contradictions. Edwin Mares' distinction between semantic and metaphysical dialetheism-the latter stating that there are true contradictions in the world-grasps the core of the matter. Mares summarises the distinction as follows:

The metaphysical dialetheist holds that there are aspects of the world (or of some possible world) for which any accurate description will contain a true contradiction. Semantic dialetheism, on the other hand, maintains that it is always possible to redescribe this aspect of the world, using different vocabulary (or perhaps vocabularies), consistently without sacrificing accuracy. (Mares 2004: 270.)

I will happily acknowledge semantic dialetheism as defined by Mares and I believe that so would most philosophers. However, it should be emphasised that the sense in which there are 'true' contradictions according to semantic dialetheism only amounts to truth in a language or model, not truth in the world-indeed, this is exactly where semantic and metaphysical dialetheism divide, because a semantic dialetheist insists that any supposed contradictions in the world can be accounted for consistently with different vocabulary. Having introduced this distinction, we might benefit from looking into a reply that Mares's article inspired from Graham Priest:

To be a metaphysical dialetheist, one must suppose that it makes sense to talk about reality itself, as opposed to what is said about it. That is, one must suppose that

I. There is an extra-linguistic reality

Next, this reality must comprise things that are propositional in some sense, or the talk of its being consistent or inconsistent would make no sense... . So we must have that

2. Reality is constituted by facts or by fact-like entities such as objects-cum-properties. Even given 2 , there is still nothing consistent or inconsistent simply in a bunch of facts. There must therefore be more to the matter than this; there must be something within the structure of facts that corresponds to negation in language. It must be the case that

3. There are polarities within facts

That is, if $\mathrm{f}^{+}$is a possible fact, say one that would make $\alpha$ true, there must be a corresponding one, $\mathrm{f}^{-}$, that would make $\neg \alpha$ true. (Priest 2006: 300.) 
As you can see, Priest is here trying to determine what sort of ontological requirements metaphysical dialetheism has. He sketches an ontology of facts and suggests that this ontology could accommodate contradictions. The crucial premise is the third one, namely the claim that there could be negative truthmakers. Priest, though, does not seem to be very interested in defending this sort of picture and adds that his In Contradiction is, in effect, neutral in regard to the semantic/metaphysical dialetheism distinction. In any case, the ontological options for accommodating contradictions are scarce. J. C. Beall (2ОоОв) has tried to promote an ontology roughly like the one Priest suggests by defending negative truthmakers. However, this looks very much like an ad hoc case, regardless of Beall's courageous defence: to uphold the idea of truthmakers having polarities Beall appeals to physics, because we have polarities in physics as well. But this is hardly relevant, for polarities of charged particles are observable by empirical means, whereas polarities within truthmakers have no such grounding. Beall (2000: 266) claims that postulating polarities for facts in metaphysics is similar to the case of postulating polarities in physics, as 'both are postulated to explain data and accommodate theory'. This seems to be his main defence against the $a d$ hoc accusation. But what sort of data is there to explain in metaphysics? It is clear that in physics we rely on empirical data, but surely there is nothing of the sort that needs explaining in metaphysics. Certainly, a metaphysical theory needs to be consistent with empirical results and it might even be able to predict future observations. However, there seems to be no empirical support whatsoever for metaphysical dialetheism and thus Beall has given us no reasons to undermine the ad hoc challenge, which has been sufficiently discussed elsewhere (cf. Molnar 2000). In comparison, all the empirical examples that we have discussed, such as the forming of atoms, corroborate a metaphysical interpretation of LNC. A more general discussion about the connection between metaphysics and empirical science would be out of place here, but I do think that metaphysics is continuous with science and should certainly take into account empirical data. In fact we might very well wish to require more than just consistency with empirical data from a theory.

\section{PRIEST'S PARADOXES CONCERNING CHANGE}

Of course, were the dialetheist to offer some further evidence suggesting that there really are contradictions in the world, then we might have to start considering feasible strategies to accommodate the idea in our ontology, even if Beall's account is unsatisfactory. Perhaps the best candidates for violations of LNC are paradoxes concerning change (cf. Priest 2006: ch. II-I2). Not every sort of change will do though. Consider our previous example: if a charged particle were to lose its charge, the instant when this change occurs is not such that there both is and is not a charge present, as clearly the causal ramifications of the electric charge either manifest or do not manifest themselves-otherwise macrophysical objects would not be possible. However, Priest, regardless of 
his supposed neutrality in terms of the semantic/metaphysical dialetheism distinction, has discussed a number of other examples (concerning change and other matters) which suggest that there might be true contradictions in the world. The best known of these is no doubt Zeno's arrow paradox, which we will discuss in the next section.

Priest starts by considering a number of everyday examples involving change and time, but these are unable to support the metaphysical dialetheist. One of these examples concerns writing a word on a paper with a pen: the pen touches the paper while the word is being written, and is lifted at the end of the word. Now, if motion is continuous, there will be an instant at which it is indeterminate whether the pen touches the paper or not, namely the instant at which the pen is lifted (Priest 2006: I60). Since we do not seem to have any reasons to decide whether the pen is touching the paper or not at this instant, we might be better off if we said that it both touches the paper and does not touch the paper-thus we have a contradiction in the world. But this does not follow: the example relies on vague terms to start with, namely 'touching the paper' is vague. This is somewhat easy to fix though, in fact Priest does it himself: "[T]here is a last point at which the electrical repulsion between my pen and the paper is equal to the weight of the pen, but no first point at which this is not the case' (ibid.). Although Priest has his concerns about this, it would appear that the paradox can be resolved; we can define 'touching the paper' in terms of the electrical repulsion between the pen and the paper (or something similar), which hardly leaves space for a contradiction in the world-at best there is confusion over language. This appears to be perfectly compatible with the semantic/metaphysical dialetheism distinction that was introduced above. If there is a way to redescribe a supposedly contradictory feature of the world in a vocabulary that preserves accuracy, then this contradictory feature does not corroborate metaphysical dialetheism, only semantic dialetheism. Priest discusses a number of other such examples, but they can all be handled in a similar manner. Consider the following example:

I am in a room. As I walk through the door, am I in the room or out of (not in) it? To emphasise that this is not a problem of vagueness, suppose we identify my position with that of my centre of gravity, and the door with the vertical plane passing through its centre of gravity. As I leave the room there must be an instant at which the point lies on the plane. At that instant am I in or out? Clearly, there is no reason for saying one rather than the other. (Priest 2006: 16I.)

Indeed, this is not a problem of vagueness in the world, but it seems to me that it is a very obvious example of vagueness concerning language. This time the question is over our definition of 'being in a room'-do we wish to define it inclusively or exclusively in regard to the doorway? Whatever we do with 
cases like this I do not see how they could be examples of contradictions in the world: the concept of 'room' is anthropocentric and because of that it will always be subject to semantic paradoxes. The reason for us lacking a specific definition for 'being in a room' is that in ordinary contexts we never need to define it as accurately as Priest here requires. If we needed to, we could very easily do that: we certainly do that when we consider whether a football is in the goal or not; it has even been suggested that a microchip should be embedded in the ball to make sure we can determine this accurately. The problem of vagueness remains, of course, but it is not the aim of this paper to solve the admittedly difficult problem of semantic vagueness, there are plenty of offers in this regard. We are here interested in whether metaphysical dialetheism is sustainable.

\section{ZENO'S ARROW}

Priest's most celebrated example of a true contradiction is Zeno's arrow paradox. It must be noted here that much of the thrust of Priest's argument relies on his particular, intrinsic view of motion, which is Hegelian in spirit ${ }^{\mathrm{IO}}$ Priest argues against the so called cinematic account of motion, according to which, say, Zeno's arrow simply occupies subsequent points in space at different times-this is all there is to its motion (cf. Priest 2006: 174). According to the cinematic account of motion at each instant of its journey the arrow is at rest and thus makes no progress, but the sum of these instants can nevertheless be greater than zero, given a sufficient number of instants (approaching infinity). Unsurprisingly, Priest is not happy with this.

What is at issue here is the nature of motion (and time) and if Priest is right, the nature of motion is fundamentally contradictory. However, we do have a number of other ways to go here, albeit all of them have their problems. Aristotle's preferred solution was to deny that time consists of indivisible instants (Aristotle 1984: 239b5-9). If the smallest instant of time is non-zero, as it apparently has to be if time does not consist of indivisibles, then motion is possible during this instant and Zeno's arrow paradox can be resolved. Another possibility (also originating from Aristotle's ideas) is to deny that there are velocities at instants - this view was later developed into the so called 'at-at' theory, which is effectively what Priest calls the cinematic account of motion. According to the 'at-at' theory, motion can be reduced to different locations at different times. This, however, seems unsatisfactory, and it is no wonder that Priest wishes to resist the account. But as E. J. Lowe (2002: 302) has pointed out, even though the measurements that we take concerning the velocity of

\footnotetext{
ro See Mortensen (2006) for further discussion; I will not discuss the details of Priest's account of motion here, as his case mainly relies on a criticism of the cinematic account of motion. I aim to evaluate the choices we have regarding motion more generally.

${ }^{11}$ For a survey of possible resolutions and the problems they face, see Arntzenius (2000).
} 
an object at a time are of course taken in terms of the locations of the object at different times, this does not mean that an object having a certain velocity fully reduces to the locations that it occupies at different times.

The fundamental problem, then, seems to be the idea of instantaneous velocity. A third way to deal with this is to understand motion as an intrinsic property, i.e. not reducible to the combination of times and locations; Arntzenius (2000) calls this the 'impetus theory'. According to this view, motion can be understood as a 'directional tendency' (cf. Lowe 2002: 243), that is, there is a difference between a stationary and a moving arrow even at an instant: a moving arrow has the tendency, the potential, if you like, to move in a certain direction. Instantaneous velocity is thus something like a dispositional property (cf. ibid., pp.302-303; see also Tooley 1988). Arntzenius (2000: section 4) discusses a number of objections to this view, but concludes that the only one that holds is an argument from ontological parsimony. Namely, to uphold the impetus theory we would have to accommodate these 'intrinsic velocities' in our ontology, as well as ensure that there is correspondence between the 'intrinsic velocity' and velocity understood as the ratio of the distance covered by an object to the period of time it takes for the object to travel that distance.

It is impossible to discuss all the implications of these different views concerning motion here, but for our purposes it is sufficient to demonstrate that the picture is certainly more complicated than a choice between the cinematic account of motion (or the 'at-at' theory) and Priest's revised Hegelian account of motion, contrary to what he seems to suggest. Ultimately we do need a complete account of motion to settle whether it might pose a problem for LNC, but this seems to be partly an empirical matter. Naturally, Priest's account of motion faces its own problems: for the very least the requirement of accommodating contradictions in our ontology, the difficulties of which we have already discussed. To this extent, Priest's account of motion and the impetus theory share the same problem, but, at least arguably, the changes required by the impetus theory are less fundamental than the ones required by Priest's theory. Accordingly, if we acknowledge the requirement for ontological parsimony (and set aside any other problems that Priest's account of motion might encounter), it would already seem that the impetus theory is preferable.

Regardless of which theory of motion is the correct one, this discussion reveals us something about the nature of the law of non-contradiction, namely, that it concerns the mind-independent reality. If the nature of motion has ramifications for LNC, then it would appear that LNC must be a metaphysical principle, as no semantic considerations of motion could settle, say, whether instantaneous velocity is possible. As noted above, this seems to be partly an empirical matter. Accordingly, metaphysical dialetheism is certainly committed to the metaphysical reading of LNC. 
THE CHALLENGE FROM QUANTUM MECHANICS

Finally, we should very briefly consider the challenge that quantum mechanics is sometimes suggested to raise for LNC. It should be noted that Priest himself does not rely on arguments based on quantum mechanics very heavily, although he does entertain a rather speculative theory in terms of the possible explanatory work that the Hegelian account of motion might be able to do in regard to the uncertainty concerning a particle's location at a time, as suggested by certain interpretations of quantum mechanics (cf. Priest 2006: 180I8I). However, arguments from quantum mechanics which seem to suggest that there could be true contradictions in the world have been offered by others ${ }^{12}$ It is not necessary to go into the details of quantum theory here, for the details are controversial in any case. What matters is that there are interpretations of quantum mechanics which imply that the reality might be in violation of the law of non-contradiction, such as the Copenhagen interpretation, and there are ones which imply the opposite, such as the Bohmian interpretation. In other words, the jury is out on the interpretation of quantum mechanics and at the moment any arguments relying on either interpretation are hardly conclusive. Presently, it is not even entirely clear that quantum mechanics can be understood to pose a challenge to LNC. Furthermore, even if there were an interpretation of quantum mechanics which satisfactorily challenges LNC, this does not necessarily mean that the law of non-contradiction is ultimately refuted, at least if we adopt a weakened sense of the principle.

I do acknowledge the theoretical fallibility of even such fundamental principles as LNC, but, quite possibly, even if the characterisation of the principle suggested above fails, a weakened version of the principle might still hold. This seems very plausible because the macrophysical world clearly is consistent. Thus, whatever the story about the microphysical involves, one of its implications is that we have consistency on the level of the macrophysical, that is, the law of non-contradiction is true at least in the sense that it is implied by the deep structure of the world, even if it would emerge from inconsistency. Accordingly, we might only have to revise the principle to ensure that it would be compatible with the inconsistency of the microphysical. This would of course be a concession to the paraconsistent logician: the metaphysical interpretation of LNC would not hold in the strong form that I have defended if it turned out that we have microphysical inconsistency. But I wish to be able to say something even in this case, namely that the metaphysical interpretation of LNC would still be useful, even if incorrect on the microphysical level, given consistency on the macrophysical level. This is, however, a very speculative point: it seems that the interpretation of quantum mechanics is fully open. So this line of thought is merely a fall-back position.

\footnotetext{
${ }^{12}$ See for instance Bueno \& Colyvan (2004); their main argument is against apriorism in logic, but they do emphasise the incompatibility of classical logic and standard quantum mechanics. The discussion is of course derived from Putnam (I979).
} 
In any case, we are still talking about the mind-independent reality. The idea is that reality as a whole might still be considered to be subject to LNC. Be it as it might that we would be ignorant about the method by which consistency on the macrophysical level could emerge from microphysical inconsistency, we would still have all the same reasons to think that the macrophysical world is consistent regardless of our thoughts and concepts concerning it. In a somewhat analogous manner we still rely on classical mechanics in most connections, even though, strictly speaking, it is false. In this weakened sense, LNC could even be compatible with metaphysical dialetheism, but currently we have no reasons to give up the full-blown version of LNC. Thus, all this is speculative; we have seen that all the arguments against the truth of the law of non-contradiction understood as a metaphysical principle based on current information are dubious at best. Unless further information emerges, I contend that metaphysical dialetheism is false.

\section{ONE TRUE LOGIC?}

I would like to conclude by considering one important ramification of this account. It would appear that if LNC is indeed a metaphysical principle, as has been extensively argued, then metaphysics may claim priority over logic. What I mean is that if principles that are usually considered as 'logical', such as LNC, are grounded in metaphysics, then we have good reasons to think that logic in general is grounded in metaphysics. Perhaps there are no such things as 'logical principles', as we might be able to ground them all in metaphysics. I wish to suggest that what we usually call 'logical principles', such as LNC, are perhaps a sub-category of metaphysical principles.

This suggests, among other things, that plausibly there can be only one true logic, that is, only one logic which fully corresponds with reality. This naturally follows from the idea that reality is one and undivided, that is, reality has a rigid structure and this structure can be described with a single formal system, if it can be described so at all. We may of course have different logics that describe reality, but they must be consistent and translatable to a single logic. Naturally it is not very straight-forward to determine what this one true logic is (and this has to be a piecemeal process in any case), in fact we can probably never fully characterise it. It is also important to see that this does not undermine the value of different logical frameworks, and we certainly do have a number of internally consistent logical frameworks which have interesting applications. However, we must be wary of any metaphysical implications that one might derive from these frameworks, for the relevance of these implications is determined by the framework's correspondence with reality, not by internal consistency. Accordingly, we can be logical pluralists in the sense that different logical frameworks may be internally valid and useful, but it should be noted 
that the notion of 'logical truth', if it is taken to imply truth in any logical system regardless of its correspondence with reality, has little bearing on truth in a metaphysically deep sense (cf. Beall and Restall 2006, p. IOO-IO2).

So, if metaphysics is about mapping the fundamental structure of reality, then logic, as I have described it, is about representing the results formally. When we reason about, say, matters of possibility and necessity, we are interested in the modal constraints that the structure of reality imposes on different kinds of entities. Modal logic, for instance, is metaphysically interesting only insofar as it reflects these constraints. The fact that we can prove the existence of God in $\mathrm{S}_{5}$ is not a very important result if we do not have good reasons to believe that $\mathrm{S}_{5}$ is the correct way to formalise the modal constraints in the world. A very natural idea concerning the different systems of modal logic is that they reflect the different uses of 'necessity' and 'possibility' in our language. But this leaves the question about modality in the world completely unanswered, quite like semantic dialetheism leaves the question of metaphysical dialetheism open. Surely we must have some kind of a theory of modality to be able to settle the status of different modal logics. Given the picture suggested above, there can be only one way in which matters stand in the actual world. So we cannot settle the question merely with the help of formal considerations.

In conclusion, the metaphysical status of principles such as the law of noncontradiction is a crucial, if not the crucial issue in our meta-logical considerations. I hope to have established here that LNC, at least, should be understood as a metaphysical principle. If I am right, we have some serious work to do in terms of the metaphysical status of logic.

\section{REFERENCES}

[I] ARISTOtle (1984) Metaphysics, trans. W. D. Ross, revised by J. Barnes (Princeton, NJ: Princeton University Press).

[2] Arntzenius, F. (2000) 'Are There Really Instantaneous Velocities?', The Monist Vol. 83 (2): I87-208.

[3] Beall, J. C. (2000A) 'Is the observable world consistent?', Australasian Fournal of Philosophy 78 (I): II3-II8.

[4] Beall, J. С. (200ов) 'On Truthmakers for Negative Truths', Australasian Fournal of Philosophy 78 (2): 264-268.

[5] BEALL, J. C. (2004) 'Introduction: At the Intersection of Truth and Falsity', in Priest, G., BeALl, J. C. and ARMOUR-GARb, B. (Eds.) The Law of Non-Contradiction (Oxford: Clarendon Press), pp. I-I9.

[6] Beall, J. C. and colyvan, M. (200I) 'Looking for contradictions', Australasian fournal of Philosophy 79 (4): 564-569. 
[7] Beall, J. C. and Restall, G. (2006) Logical Pluralism (Oxford: Clarendon Press).

[8] BEALL, J. C. and PRIEST, G. (2007) 'Not so deep inconsistency: a reply to Eklund', Australasian fournal of Logic 5: 74-84.

[9] bueno, O. and colyvan, M. (2004) 'Logical Non-Apriorism and the "Law" of Non-Contradiction', in PRIEST, G., BEALL, J. C. and ARMOURGARB, B. (Eds.) The Law of Non-Contradiction (Oxford: Clarendon Press), pp. $156-175$.

[io] Dutilh novaes, C. (2008) 'Contradiction: the Real Philosophical Challenge for Paraconsistent Logic', in BÉZIAU, J. Y., CARNI ELLI, w., and GABBAY, D. (Eds.), Handbook of Paraconsistency, London: College Publications.

[II] E KLund, M. (2002A) 'Inconsistent Languages', Philosophy and Phenomenological Research 64 (2): 26I-275.

[I2] EkLUnd, M. (2002B) 'Deep Inconsistency' Australasian fournal of Philosophy 80 (3): 32I-33I.

[13] EkLund, M. (2008) 'Reply to Beall and Priest', Australasian fournal of Logic 6: 94-106.

[I4] Hyde, D. and Colyvan, M. (2008) 'Paraconsistent Vagueness: Why Not?', Australasian Journal of Logic $6:$ I08-I2I.

[15] LOwE, E. J. (2002) A Survey of Metaphysics (Oxford: Oxford University Press).

[i6] mares, E. D. (2004) 'Semantic Dialetheism', in Priest, G., BeAll, J. C. and armour-Garb, в. (Eds.) The Law of Non-Contradiction (Oxford: Clarendon Press), pp. 264-275.

[17] MOlnar, G. (2000) 'Truthmakers for Negative Truths', Australasian fournal of Philosophy 78 (I): 72-86.

[I8] Mortensen, C. (2006) 'Change', The Stanford Encyclopedia of Philosophy (Winter 2006 Edition), EDWARD N. ZaLTa (ed.), URL = http://plato. stanford.edu/archives/win2006/entries/change/.

[ig] politis, v. (2004) Routledge Philosophy GuideBook to Aristotle and the Metaphysics (London and New York: Routledge).

[20] PRICE, H. (I990) 'Why “Not"?', Mind 99: 22I-238.

[2I] PRIEST, G. (1999) 'Perceiving contradictions', Australasian fournal of Philosophy 77 (4): 439-446. 
[22] Priest, G. (2006) In Contradiction: A Study of the Transconsistent, $2^{\text {nd }}$ expanded ed. (Oxford: Clarendon Press).

[23] putnam, H. (1979) 'The Logic of Quantum Mechanics', in Mathematics, Matter and Method: Philosophical Papers, $2^{\text {nd }}$ edn. (Cambridge: Cambridge University Press), pp. 174-197.

[24] SLATER, B. H. (1995) 'Paraconsistent Logics?' Journal of Philosophical Logic 24: $45^{\mathrm{I}-454}$.

[25] tooley, M. (1988) 'In Defense of the Existence of States of Motion', Philosophical Topics 16: 225-54. 
The Australasian fournal of Logic (ISSN I448-5052) disseminates articles that significantly advance the study of logic, in its mathematical, philosophical or computational guises. The scope of the journal includes all areas of logic, both pure and applied to topics in philosophy, mathematics, computation, linguistics and the other sciences.

Articles appearing in the journal have been carefully and critically refereed under the responsibility of members of the Editorial Board. Only papers judged to be both significant and excellent are accepted for publication.

The journal is freely available at the journal website at

$$
\text { http://www.philosophy.unimelb.edu.au/ajl/ }
$$

All issues of the journal are archived electronically at the journal website.

Subscriptions Individuals may subscribe to the journal by sending an email, including a full name, an institutional affiliation and an email address to the managing editor atajl-editors@unimelb.edu.au. Subscribers will receive email abstracts of accepted papers to an address of their choice. For institutional subscription, please email the managing editor atajl-editors@unimelb.edu.au

Complete published papers may be downloaded at the journal's website at http: //www.philosophy.unimelb.edu.au/ajl/ The journal currently publishes in pdf format.

Submission The journal accepts submissions of papers electronically. To submit an article for publication, send the $\mathrm{LT}_{\mathrm{E}} \mathrm{X}$ source of a submission to a member of the editorial board. For a current list of the editorial board, consult the website.

The copyright of each article remains with the author or authors of that article. 